\title{
Guide canadien d'immunisation : Mise à jour du chapitre "Anaphylaxie et autres réactions aiguës après la vaccination »
}

\author{
Natalie Dayneka ${ }^{1,2}$, Christina Jensen ${ }^{3}$, Kyla Hildebrand ${ }^{4,5}$ au nom du Comité consultatif national de \\ l'immunisation (CCNI)*
}

\section{Résumé}

Contexte : Le Guide canadien d'immunisation est une ressource exhaustive sur l'immunisation à I'intention des professionnels de la santé et des décideurs des programmes de vaccination. Il est élaboré à partir des recommandations fondées sur des données probantes du Comité consultatif national de l'immunisation (CCNI). Le Groupe de travail sur l'innocuité des vaccins du CCNI est composé de membres du CCNI, de membres de liaison et d'experts externes. L'Organisation mondiale de l'allergie recommande maintenant que les antihistaminiques ne soient pas utilisés dans le traitement initial de l'anaphylaxie. La mise à jour du chapitre a également été utilisée pour fournir des renseignements et des éclaircissements supplémentaires à plusieurs tableaux du chapitre.

Méthodes : En mettant à jour le guide d'anaphylaxie du Guide canadien d'immunisation, le Groupe de travail sur l'innocuité des vaccins a effectué une analyse environnementale, un examen de la documentation pertinente et consulté des experts internationaux ainsi que canadiens et des sociétés professionnelles.

Résultats : II n'est plus recommandé d'utiliser le chlorhydrate de diphénhydramine comme traitement complémentaire dans la gestion de l'anaphylaxie dans un milieu communautaire. Voici d'autres changements notables apportés au chapitre : 1) réintitulé : « Anaphylaxie et autres réactions aiguës après la vaccination »; 2 ) inclusion de nouveaux tableaux : "Caractéristiques principales de l'anaphylaxie et de la syncope vasovagale » et " Signes et symptômes de l'anaphylaxie »; et 3) tableaux mis à jour : "Trousse de gestion de l'anaphylaxie : produits recommandés » et " Dosage de la solution intramusculaire ÉPINÉPHrine 1:1000 (1 mg/ml), selon l'âge ou le poids ».

Conclusion : Le chapitre du Guide canadien d'immunisation mis à jour donne aux fournisseurs de soins de santé une plus grande clarté dans la reconnaissance et la gestion de l'anaphylaxie dans les milieux communautaires. Le tableau posologique intramusculaire actualisé de l'épinéphrine aidera à une administration optimale de l'épinéphrine, tandis que la directive révisée contre l'utilisation du chlorhydrate de diphénhydramine empêchera son stockage inutile en préparation de cliniques de vaccination de masse potentielles liées à la pandémie de coronavirus de 2019.
Cette oeuvre est mise à la disposition selon les termes de la licence internationale Creative Commons Attribution 4.0

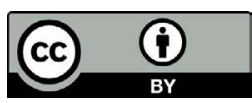

Affiliations

1 Président du Groupe de travail sur la sécurité des vaccins du $\mathrm{CCNI}$

${ }^{2}$ Département de pharmacie, Hôpital pour enfants de l'Est de I'Ontario, Ottawa, ON

${ }^{3}$ Centre de l'immunisation et des maladies respiratoires infectieuses, Agence de la santé publique du Canada, Ottawa, ON

${ }^{4}$ Division de l'allergie et de l'immunologie, Département de pédiatrie, Faculté de médecine, Université de la Colombie-Britannique, Vancouver, BC

${ }^{5}$ Institut de recherche de l'Hôpital pour enfants de la Colombie-Britannique, Vancouver, BC

\section{*Correspondance :}

phac.naci-ccni.aspc@canada.ca

Citation proposée : Dayneka N, Jensen C, Hildebrand KJ, au nom du Comité consultatif national de l'immunisation (CCNI). Guide canadien d'immunisation : Mise à jour du chapitre " Anaphylaxie et autres réactions aiguës après la vaccination ». Relevé des maladies transmissibles au Canada 2020;46(11/12):432-4.

https://doi.org/10.14745/ccdr.v46i1112a04f

Mots-clés : Comité consultatif national de l'immunisation, CCNI, Guide canadien d'immunisation, GCl, anaphylaxie, directives

\section{Introduction}

Le Guide canadien d'immunisation est une ressource exhaustive sur l'immunisation à l'intention des professionnels de la santé et des décideurs des programmes de vaccination. Il est élaboré à partir des recommandations fondées sur des données probantes du Comité consultatif national de l'immunisation (CCNI). 
Les recommandations du CCNI sont élaborées par des groupes de travail spécifiques. Le Groupe de travail sur l'innocuité des vaccins du CCNI est composé de membres du CCNI, de membres de liaison et d'experts externes. Il est chargé de fournir des conseils sur les révisions des chapitres de la partie 2 (Innocuité des vaccins) qui portent sur la surveillance de l'innocuité des vaccins au Canada, les contre-indications et les précautions, ainsi que sur l'évaluation et la gestion de I'anaphylaxie.

Depuis la dernière mise à jour du chapitre, l'Organisation mondiale de l'allergie a révisé ses recommandations sur la gestion de l'anaphylaxie dans la collectivité. L'Organisation mondiale de l'allergie recommande maintenant que les antihistaminiques ne soient pas utilisés dans le traitement initial de l'anaphylaxie (1). La mise à jour du chapitre a également été utilisée pour fournir des renseignements et des éclaircissements supplémentaires à plusieurs tableaux du chapitre.

\section{Méthodes}

En mettant à jour le guide d'anaphylaxie du Guide canadien d'immunisation (2), le Secrétariat du CCNI a effectué une analyse environnementale, un examen de la documentation pertinente et consulté des experts internationaux et canadiens et des sociétés professionnelles. Le Groupe de travail sur l'innocuité des vaccins a examiné et discuté les éléments de preuve suivants :

- La position optimale pour les personnes ayant une réaction anaphylactique

- Les recommandations, lignes directrices et pratiques canadiennes et internationales concernant le site optimal et la posologie de l'administration de l'épinéphrine

- L'utilisation du chlorhydrate de diphénhydramine (Benadry ${ }^{\mathrm{MD}}$ ) dans la gestion de l'anaphylaxie dans la collectivité

Les lignes directrices mises à jour, y compris l'élimination de la recommandation de traitement complémentaire et le tableau sur la dose d'épinéphrine par âge ou poids, ont été présentées au $\mathrm{CCNI}$ pour approbation.

\section{Résultats}

Dans le cas de l'anaphylaxie, le Groupe de travail sur l'innocuité des vaccins a clarifié sa recommandation de placer les personnes sur le dos (supine) et d'élever leurs extrémités inférieures. Tant que la réaction anaphylactique n'est pas complètement gérée, le vacciné doit rester dans cette position semi-couchée, car la létalité peut survenir rapidement en raison du syndrome veine cave vide/ventricule vide (si le vacciné se tient ou s'assoit subitement).

Le Groupe de travail sur l'innocuité des vaccins a confirmé qu'il existe de bonnes données à l'appui de la conclusion que ni le deltoïde ni les muscles glutineux ne devraient être le site de l'administration de l'épinéphrine. L'épinéphrine devrait toujours être fournie de manière intramusculaire dans la partie antérolatérale médiane de la cuisse (vastus lateralis), étant donné qu'elle a un approvisionnement sanguin important.

À la suite de l'examen des données de l'Organisation mondiale de l'allergie, le Groupe de travail sur l'innocuité des vaccins ne recommande plus l'utilisation d'antihistaminiques comme traitement complémentaire dans la gestion de l'anaphylaxie dans un milieu communautaire. L'utilisation de la thérapie d'appoint n'a pas été jugée appropriée dans le milieu communautaire, puisque le rôle du fournisseur de vaccins dans la gestion de l'anaphylaxie après l'immunisation consiste principalement à gérer le patient (en fournissant l'épinéphrine et la surveillance) jusqu'à l'arrivée des soins d'urgence.

Le Groupe de travail sur l'innocuité des vaccins a également fourni des conseils supplémentaires sur la posologie intramusculaire de l'épinéphrine selon l'âge, étant donné que de nombreux fournisseurs de vaccins n'ont pas accès au poids d'un client (e.g. il n'y a peut-être pas de balance dans une pharmacie, une clinique d'immunisation de masse, une clinique de santé publique, etc.). Bien que la documentation appuyant l'administration d'épinéphrine par auto-injecteur à des nourrissons de moins de $10 \mathrm{~kg}$ ait été jugée limitée, le Groupe de travail sur l'innocuité des vaccins a estimé que les avantages de l'utilisation d'épinéphrine chez ces personnes l'emportent sur les risques, même si cette utilisation serait considérée comme non indiquée sur l'étiquette au Canada.

Le Groupe de travail sur l'innocuité des vaccins a mis à jour le tableau posologique de l'épinéphrine, qui est utilisée depuis juin 2013 et a été initialement mise au point par la coalition d'action pour l'immunisation. Le tableau posologique révisé de l'épinéphrine a été adapté à partir de l'algorithme d'anaphylaxie pédiatrique de Partage des connaissances pédiatriques en urgence (TREKK) $(3,4)$, qui est un programme canadien visant à améliorer les soins d'urgence pédiatrique. De plus, les tranches d'âge pour l'épinéphrine dosée ont été sélectionnées dans le Australian Immunization Handbook (5), car elles correspondaient bien aux bandes de poids du tableau TREKK. Ce nouveau tableau adapté (Tableau 4 : Dosage de l'ÉPINÉPHrine intramusculaire 1:1000 [1 mg/ml], selon l'âge ou le poids) a été examiné et approuvé par des experts de la Société canadienne d'allergie et d'immunologie clinique en août 2020.

Voici d'autres changements notables apportés au chapitre :

- Nouveau titre: Auparavant, «Réactions précoces au vaccin incluant l'anaphylaxie », maintenant « Anaphylaxie et autres réactions aiguës après la vaccination »

- Inclusion d'un nouveau tableau : «Tableau 1: Caractéristiques principales de l'anaphylaxie et de la syncope vasovagale » 
- Inclusion d'un nouveau tableau : «Tableau 2 : Signes et symptômes de l'anaphylaxie "

- Tableau mis à jour : «Tableau 3 : Trousse de gestion de I'anaphylaxie : objets recommandés »

\section{Conclusion}

Le chapitre du Guide canadien d'immunisation mis à jour donne aux fournisseurs de soins de santé une plus grande clarté dans la reconnaissance et la gestion de réactions anaphylactiques dans les milieux communautaires. Le développement du nouveau tableau de posologie intramusculaire d'épinéphrine aidera à une administration optimale de l'épinéphrine, de nouvelles recommandations sur I'utilisation du chlorhydrate de diphénhydramine empêcheront son stockage inutile en préparation de cliniques de vaccination de masse potentielles liées à la pandémie de coronavirus de 2019.

\section{Déclaration des auteurs}

C. J. - Rédaction, ébauche originale, révision, édition

N. D. - Examen, modification

K. H. - Révision, modification

Le chapitre du Guide canadien d'immunisation, « Anaphylaxie et autres réactions aiguës après la vaccination " a été révisé par le Groupe de travail sur l'innocuité des vaccins du Comité consultatif national de l'immunisation (CCNI) et approuvé par le CCNI.

\section{Intérêts concurrents}

Aucun.

\section{Remerciements}

Membres du Groupe de travail sur l'innocuité des vaccins : N. Dayneka (présidente du GT), C. Jensen (Agence de la santé publique du Canada [l'Agence]), K. Hildebrand, V. Dubey, O. Baclic (I'Agence), J. Gallivan, M. Salvadori, A. Pham-Huy, H. Anyoti (l'Agence), K. Johnson (l'Agence), B. Seifert (expert externe), K. Top (expert externe), B. Law (expert externe), W. Alqurashi (expert externe)

Membres du CCNI : C. Quach (Présidente), S. Deeks (Vice-Présidente), N. Dayneka, P. De Wals, V. Dubey, R. Harrison, K. Hildebrand, C. Rotstein, M. Salvadori, B. Sander, N. Sicard et S. Smith

Représentants de liaison : L. M. Bucci (Association canadienne de santé publique), E. Castillo (Société des obstétriciens et gynécologues du Canada), A. Cohn (Centers for Disease Control and Prevention, États-Unis), J. Emili (Collège des médecins de famille du Canada), M. Naus (Comité canadien d'immunisation), D. Moore (Société canadienne de pédiatrie) et $A$. Pham-Huy (Association de microbiologie médicale et de maladies infectieuses Canada)

Représentants d'office: J. Gallivan (Direction des produits de santé commercialisés, Santé Canada [SC]), E. Henry (Centre de I'immunisation et des maladies respiratoires infectieuses [CIMRI], l'Agence), M. Lacroix (Groupe consultatif sur l'éthique en santé publique, I'Agence), J. Pennock (CIMRI, I'Agence), R. Pless (Direction des produits biologiques et des thérapies génétiques, SC), G. Poliquin (Laboratoire national de microbiologie, l'Agence) T. Wong (Direction générale de la santé des Premières Nations et des Inuits, Services autochtones Canada)

Le Comité consultatif national de l'immunisation reconnaît et apprécie les contributions de $\mathrm{D}^{\mathrm{r}} \mathrm{H}$. Kim, $\mathrm{D}^{\text {re }} \mathrm{J}$. Upton, $\mathrm{D}^{\text {re }} \mathrm{S}$. Redpath, $\mathrm{D}^{\mathrm{r}} \mathrm{M}$. Tunis et $\mathrm{C}$. Mauviel à cette révision du chapitre.

\section{Financement}

Le travail du Comité consultatif national de l'immunisation est appuyé par l'Agence de la santé publique du Canada.

\section{Références}

1. Simons FE, Ebisawa M, Sanchez-Borges M, Thong BY, Worm M, Tanno LK, Lockey RF, El-Gamal YM, Brown SG, Park HS, Sheikh A. 2015 update of the evidence base: world Allergy Organization anaphylaxis guidelines. World Allergy Organ J 2015;8(1):32. DOI PubMed

2. Comité consultatif national de l'immunisation. Guide canadien d'immunisation. Anaphylaxis and other acute reactions following vaccination (chapitre) Ottawa (ON) : ASPC; 2020. https://www.canada.ca/fr/sante-publique/ services/publications/vie-saine/guide-canadie n-immunisation-partie-2-innocuite-vaccins/page-4-innoc uite-vaccins-effets-secondaires-suivant-immunisation.html

3. Translating Emergency Knowledge for Kids (TREKK). Pediatric anaphylaxis algorithm. Dec 2018. Version 1.1 (accédé 2020-06-28). https://trekk.ca/system/assets/ assets/attachments/339/original/2018-12-10_Anaphylaxis_ algorithm_v_1.1.pdf?1545083235

4. Translating Emergency Knowledge for Kids (TREKK). Bottom Line Recommendations: Anaphylaxis. December 2018, Version 1.2 (accédé 2019-07-09). https://trekk.ca/ system/assets/assets/attachments/338/original/2018-12-14_ Anaphylaxis_BLR_version_1.2.pdf?1545083199

5. Australian Government Department of Health. Australian Technical Advisory Group on Immunisation. Australian Immunisation Handbook. Canberra, Australia: ATAGI; 2018. https://immunisationhandbook.health.gov.au/ 